\section{Light processable starch hydrogels}

\author{
Camilla Noè, ${ }^{1}$ Chiara Tonda-Turo, ${ }^{2}$ \\ Annalisa Chiappone, ${ }^{1}$ \\ Marco Sangermano, ${ }^{1}$ \\ Minna Hakkarainen² \\ 1Politecnico di Torino, Dipartimento di \\ Scienza Applicata e Tecnologia, \\ University of Torino, Turin; \\ 2Department of Fibre and Polymer \\ Technology, KTH Royal Institute of \\ Technology, Stockholm, Sweden
}

\begin{abstract}
Light processable hydrogels were successfully fabricated by utilizing maize starch as raw material. Increasing the starch content from 10 to $15 \mathrm{wt} \%$ increased the compressive stiffness from 13 to $20 \mathrm{kPa}$, which covers the stiffness of different body tissues.
\end{abstract}

\section{Introduction}

Hydrogels are three-dimensional polymeric networks with the ability of retaining large amount of water within their structures without dissolving. Thanks to their hydrophilicity, hydrogels can be utilized in a broad range of sectors including pharmaceutical and medical. ${ }^{1}$ Starch based hydrogels are gaining attractiveness due to their renewability and degradability. Moreover, starch-based hydrogels are partially able to mimic the natural cells aqueous environment. ${ }^{2}$ Photocuring techniques are green and highly efficient for crosslinking polymer resin with photo-curable functional groups. They have many advantages with respect to the thermocuring processes such as absence of Volatile Organic Compound (VOC) emissions, high curing speed, reduced energy consumption, and no heat requirements. 3 $3 \mathrm{D}$ printing by digital light processing has gained significant attention as a tool for designing biomedical scaffolds. ${ }^{4}$ In this context, functionalizedstarch able to photo-crosslink in water to be exploited as photo-curable and 3D-printable hydrogel precursor would be of high interest. Therefore, starch was methacrylated followed by evaluation of light processability by photo-curing in a mould or by Digital Light Processing (DLP) 3D printing. Furthermore, the light processed hydrogels were characterized for mechanical properties and cytotoxicity.

\section{Materials and Methods}

High-amylose Hylon VII maize starch $(70 \%$ amylose $)$ was obtained from Ingredion, Goole,

UK. Methacrylic anhydride (MA), triethylamine (TEA) (>99\%), dimethyl sulfoxide (DMSO) (ACS reagent P99.9\%), ethanol absolute, and bis(acyl)phosphane oxi lithium phenyl-2,4,6-trimethylbenzoyl phosphinate (LAP) were purchased from Sigma Aldrich (Milan, Italy).

\section{Results}

Maize starch was methacrylated by a simple reaction with methacrylic anhydride in presence of triethylamine (nucleophilic catalyst). A scheme of the reaction is reported in the Figure 1. The success of the methacrylation reaction was confirmed by ${ }^{1} \mathrm{H}-\mathrm{NMR},{ }^{13} \mathrm{C}-\mathrm{NMR}$, and FTIR spectroscopy. The obtained degree of substitution (DOS) for the hydroxyl groups was 0.08 which can be translated into one methacrylated alcohol-group in every fourth glucose rings. Three different formulations containing 5,10 and $15 \mathrm{wt} \%$ of methacrylated starch (MA-Starch) in water were photocured with $1 \mathrm{phr}$ of LAP. The reactivity of those formulations was evaluated by a photo-rheology test. Both the formulations containing 10 and $15 \mathrm{wt} \%$ of MA-Starch started reacting immediately and reached a plateau after 90 seconds, while the $5 \mathrm{wt} \%$ shows a delay in the curing process and reached a plateau after $120 \mathrm{sec}-$ onds. This can be explained by the low starch concentration in water, which in combination with the overall low DOS decreased the reaction velocity.

The mechanical properties of the hydrogels were investigated by a compression test. The obtained Young's modulus values (Ec) are: $12 \mathrm{kPa}$ for $10 \mathrm{wt} \% \mathrm{MA}-\mathrm{Starch}$ and
Correspondence: Minna Hakkarainen, Department of Fibre and Polymer Technology, KTH Royal Institute of Technology, Stockholm, Sweden.

E-mail: minna@kth.se

Key words: Hydrogels; UV-curing; starch.

Disclosures: Authors have nothing to disclose.

Conference presentation: This paper was presented at the Third Centro 3R Annual Meeting - L'era delle 3R: modelli in silico, in vitro e in vivo per promuovere la ricerca traslazionale 30 September - 1 October 2021, Evento online organizzato dal Politecnico di Torino.

Received for publication: 9 July 2021.

Accepted for publication: 7 September 2021.

This work is licensed under a Creative Commons Attribution NonCommercial 4.0 License (CC BY-NC 4.0).

CCopyright: the Author(s), 2021

Licensee PAGEPress, Italy

Biomedical Science and Engineering 2021; 4(s1):184 doi:10.4081/bse.2021.184

$20 \mathrm{kPa}$ for $15 \mathrm{wt} \% \mathrm{MA}-\mathrm{Starch}$. The simple increase of the MA-Starch concentration in water increased the compressive stiffness of the hydrogels. This range covers the stiffness of different native body tissues, which is promising for utilization of the hydrogels in tissue engineering applications.

Human fibroblast cells were cultured on the supernatants from the three hydrogel formulations. The cell viability was very similar to the control, indicating no negative effects from the methacrylation, photocrosslinking reaction and LAP photo-initiator. The fast photo-curing reactivity of the formulations and the obtained mechanical properties indicated that the MA-Starch could be suitable photo-curable resin for the vat $3 \mathrm{D}$ printing technology. The $3 \mathrm{D}$ print-
Maize Starch
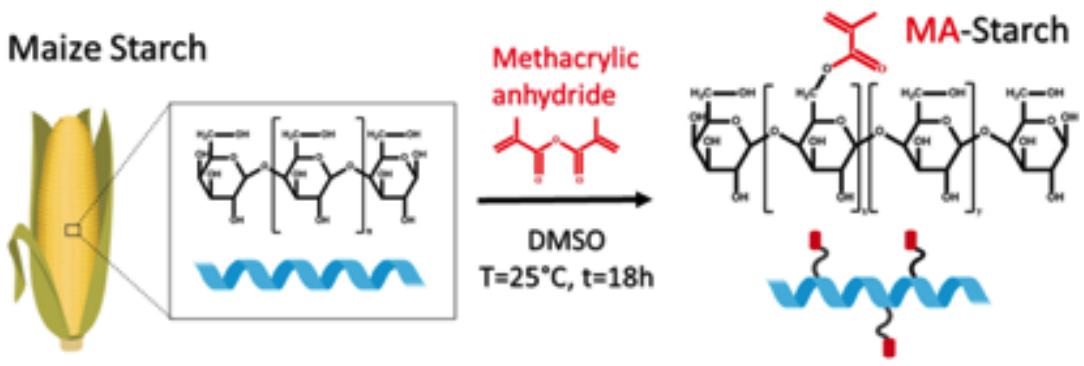

Figure 1. Schematic illustration of starch methacrylation. 
ability with DLP printing of $10 \mathrm{wt} \%$ MAStarch was also investigated since this concentration showed a compression Young modulus close to the elasticity of muscle tissue $(12 \mathrm{kPa}) .5$ A honeycomb structure was designed and successfully printed using a DMEM-starch solution instead of waterstarch solution showing that millimeterthick wall structures could be built from this formulation (Figure 2).

\section{Discussion and Conclusions}

Methacrylated maize starch was successfully light processed to hydrogels either by photo-curing with UV-lamp or by DLP 3D-printing directly from aqueous solution. First, starch was methacrylated and the reaction was confirmed by ${ }^{1} \mathrm{H}-\mathrm{NMR},{ }^{13} \mathrm{C}$ NMR, and FTIR spectroscopy. The methacrylated starch was dispersed in water at different concentration in the presence of LAP photo-initiator. Photo-rheology confirmed rapid in-situ gel formation by following the evolution of elastic storage mod-
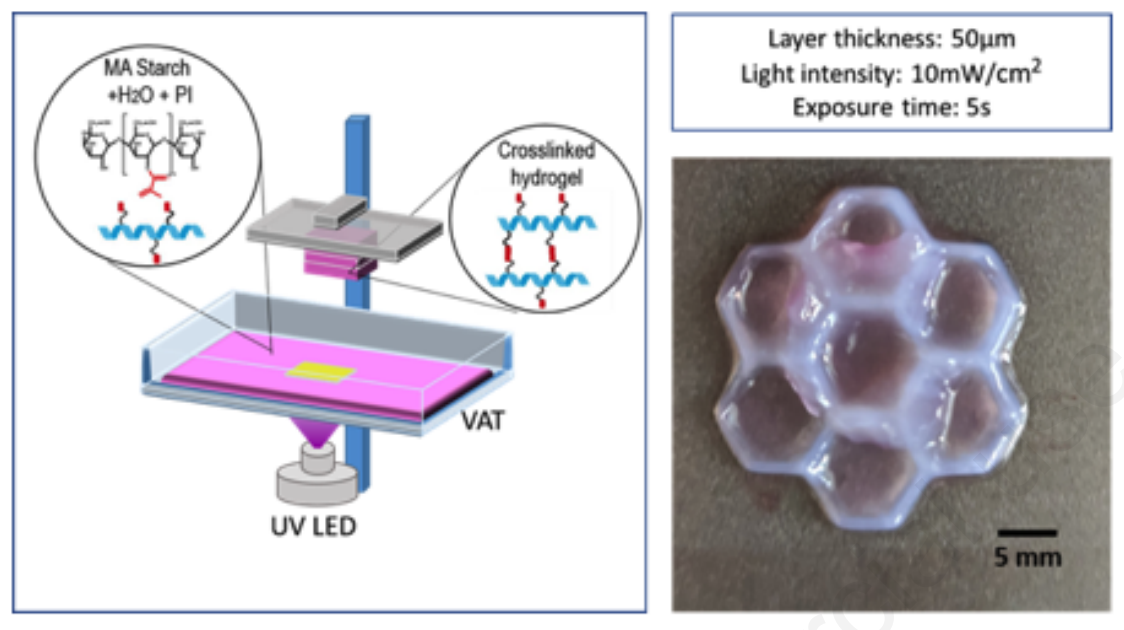

Figure 2. Left) 3D DLP printed hydrogels, right) complex flower geometry [ $24 \mathrm{~mm} \times 24$ $\mathrm{mm} \times 4 \mathrm{~mm}]$. ulus G' with time. The supernatants of the methacrylated starch-based photocrosslinked hydrogels did not exhibit cytotoxicity against human $\mathrm{BJ}$ fibroblast cells in any of the three tested hydrogel concentrations. Finally, the 3D printability of $10 \mathrm{wt} \%$ MA-Starch was successfully demonstrated. These findings are promising for future applications of light processed starch in tissue engineering and as cell carriers.

\section{References}

1. Van Vlierberghe S, Dubruel P, Schacht E. Biopolymer-based hydrogels as scaffolds for tissue engineering applications: a review. Biomacromolecules 2011;12:1387-408.

2. Piluso S, Labet M, Zhou C, et al. Engineered three-dimensional microenvironments with starch nanocrystals as cell-instructive materials. Biomacromolecules 2019;20:3819-30.

3. Malburet S, Di Mauro C, Noè C, et al. Sustainable access to fully biobased epoxidized vegetable oil thermoset materials prepared by thermal or UVcationic processes. RSC Adv 2020;10: 41954-66

4. Chimene D, Kaunas R, Gaharwar AK. Hydrogel bioink reinforcement for additive manufacturing: a focused review of emerging strategies. Adv Mater 2020;32:1902026.

5. Han F, Zhu C, Guo Q, Yanga H, Li B. Cellular modulation by the elasticity of biomaterials. J Mater Chem B 2016;4: 9-26. 